\title{
Emergências em pacientes com doença de Chagas na cidade de Buenos Aires, Argentina
}

\author{
Emergencies in patients with Chagas' disease \\ in Buenos Aires city, Argentina \\ Sergio Auger ${ }^{1,}$ Rubén Storino ${ }^{2}$, Oscar Iglesias Ordoñez ${ }^{2}$, María Inés Urrutia ${ }^{2}$, \\ Mariana Sanmartino ${ }^{2}$, Dardo Romero² e Miguel $\mathrm{Jörg}^{3^{*}}$
}

\begin{abstract}
Resumo O objetivo do trabalho foi avaliar as causas mais freqüentes de emergências em pacientes com doença de Chagas. Entre janeiro de 1998 e janeiro de 1999, foram incluídos num estudo prospectivo, indivíduos com doenca de Chagas que ingressaram no pronto socorro do Hospital Santojanni da Cidade de Buenos Aires. Para a análise estatística, utilizou-se o teste de $X^{2}$ com correção de Yates. De um total de 1.680 pacientes ingressados, houve 95 (6\%) com sorologia reativa para doença de Chagas. Em 31 indivíduos, a causa de ingresso foi síncope, em 28 insuficiência cardíaca, em 18 eventos coronários agudos, em 5 acidentes vasculares cerebrais, em 3 hipertensão arterial com edema agudo de pulmão e em 2 encefalite aguda associada à SIDA. Como conclusão observou-se uma associação significativa entre: 1) presença de cardiopatia e hospitalização, 2) insuficiência cardíaca, síncope, encefalite aguda e mortalidade, 3) progresso de cardiopatia e mortalidade e 4) lugar de procedência e mortalidade.
\end{abstract}

Palavras-chaves: Doença de Chagas. Emergência. Hospitalização. Cardiopatia. Mortalidade.

\begin{abstract}
The objective of this work was to evaluate the most frequent causes of emergency in patients with Chagas disease. Between January 1998-January 1999, individuals with Chagas disease inside the shockroom of Santojanni Hospital in Buenos Aires city, were included in a prospective study. For the statistical analysis, $X^{2}$ test with Yates correction has been used. From a total of 1.680 patients entered, there were $95(6 \%)$ with reactive serology for Chagas disease. In 31 individuals the enter cause was syncope, in 28 cardiac insufficiency, in 18 acute coronary events, in 5 stroke, in 3 acute edema of hypertensive lung and in 2 acute encephalitis associated to AIDS. In conclusion, significant association has been observed between: 1) presence of cardiopathy and hospitalization, 2) cardiac insufficiency, syncope, acute encephalitis and mortality, 3) cardiopathy development and mortality and 4) origin place and mortality.
\end{abstract}

Key-words: Chagas' disease. Emergency. Hospitalization. Cardiopathy. Mortality.

A doença de Chagas é uma afecção parasitária dos tecidos e do sangue produzida pelo protozoário flagelado Trypanosoma cruzi, que se aninha e reproduz nos tecidos. Esta afecção é transmitida por insetos hemípteros (triatomíneos), sendo o mais freqüente no ciclo nosogênico (na Argentina), o Triatoma infestans é adaptado ecologicamente à residência humana. Epidemiologicamente, esta doença depende da presença de quatro elementos: 1) o agente etiológico, dado pelo T. cruzi; 2) o vetor, representado pelo triatomíneo; 3 ) o hóspede sensível à infecção com T. cruzi e 4) condições para sua domicialização (moradia) 7192324 . Na Argentina, estas condições são encontradas, fundamentalmente, no interior do país, na área rural. Porém, devido às migrações internas e imigrações dos países endêmicos vizinhos, para as grandes urbes e do crescimento paralelo da pobreza nas zonas urbanas, observou-se nos últimos anos um elevado incremento de indivíduos com sorologia positiva na cidade de Buenos Aires ${ }^{2}$. Como produto desta migração, põem-se de manifesto as formas urbanas de transmissão, principalmente através da hemotransfusão e congênita, ainda que esta última se observa também nas áreas rurais. Segundo a

\footnotetext{
1. Hospital Público General de Agudos Francisco Santojanni, Buenos Aires, Argentina, 2. Centro de Enfermedad de Chagas de la Fundación INCALP, La Plata, Buenos Aires, Argentina. 3. Centro Médico de Mar del Plata, Buenos Aires, Argentina. * Autor falecido em novembro de 2002. Endereço para correspondência: Dr. Rubén Storino. Calle 56 Nro 715, 1900, La Plata, Provincia de Buenos Aires, Argentina. Tel: $+54221482-7012$

e-mail: camastro@isis.unlp.edu.ar

Recebido para publicação em 15/10/2001.
} 
Organização Mundial da Saúde, a doença de Chagas é uma das doenças tropicais mais comuns da América Latina, ocupando o terceiro lugar depois da febre amarela e da esquistossomose ${ }^{18}$. A prevalência estimada da infecção no continente estabelece que 90 milhões de pessoas estão em risco de contrair a doença, cerca de 24 milhões estão parasitadas e aproximadamente 6 milhões já são cardiopatas ${ }^{15}$. Na Argentina, oficialmente estimamse ao redor de 2,3 milhões de infestados (os pesquisadores das áreas rurais suspeitam de números maior) ${ }^{20}$, com aproximadamente 700.000 cardiopatas $^{2324}$.

A infecção humana não segue um protocolo estabelecido. $O$ número e a natureza dos parasitas infectantes, o momento da infecção, o estado nutricional, imunológico, as características genéticas dos seres humanos, a possibilidade de reinfecção e a existência de diversas patologias são variáveis difíceis de avaliar. O indivíduo, em geral, vai desenvolver a doença 20 a 30 anos após a entrada do parasita no organismo. Esta situação, dificulta as conclusões do seguimento clínico dos indivíduos infectados e dos fatos que intervêm na passagem da fase latente indeterminada à miocardiopatia sintomática ${ }^{24}$

A análise estatística multivariada realizada em 2.260 indivíduos chagásicos ${ }^{22}$, reunindo pacientes de vários centros de atenção dos doentes chagásicos, como o Instituto de Cardiologia da Fundação Pombo de Rodrigues (Capital Federal), o Hospital Fernández (Capital Federal), o Hospital Santojanni (Capital Federal), o Centro de Atenção Ambulatorial da Fundação INCALP (La Plata, província de Buenos Aires), todos eles assistidos pela equipe de saúde dirigida pelos Doutores Rubén Storino e Sergio Auger, confirmou maior possibilidade de dano do miocárdico nas seguintes situações: 1) pacientes de mais de 40 anos, 2) procedência de zona de alta endemicidade, especialmente de Santiago del Estero, Catamarca e La Rioja, 3) ter habitado em área endêmica durante muitos anos, com um índice diretamente proporcional ao aumento do tempo de residência, 4) o habitat endêmico com a infecção por via vetorial teria maior probabilidade na evolução para cardiopatia chagásica que a hemotransfusão e a transmissão congênita, 5) apresentar o exame físico anormal e 6) referir sintomas, especialmente de sincope e dispnéia. Sem dúvida nenhuma, são muitos os fatores de risco que se apontam como determinantes do desenvolvimento da cardiopatia chagásica na fase crônica da doença. Dentre estes fatores, também devemos mencionar o componente genético, a cepa e o zimodema do T. cruzi que interveio, a gravidade da fase aguda, os altos níveis de parasitemia em forma persistente, a reinfecção endógena diante da imunodepressão e as contínuas reinfecções por viver em zona endêmica com alta infestação. Sabe-se ademais, que o fator imunológico tem um rol importante na fisiopatogenia desta doença e inclusive na evolução para a cardiopatia ${ }^{1613}$. Observase assim que a existência dos anticorpos anti-receptores b e os anticorpos anti-receptores muscarínicos ${ }^{9} 10$ têm uma estreita relação com fenômenos funcionais, como a notável associação entre disautonomia e bradicardia. Finalmente, as complicações mais comuns, encontradas na miocardiopatia chagásica crônica, são presença de aneurismas ventriculares, arritmias ventriculares graves, transtornos da condução avançados, insuficiência cardíaca congestiva, tromboembolismo pulmonar ou sistêmico e morte súbita ${ }^{23} 24$.

Os objetivos deste trabalho foram: a) determinar a prevalência de internação de pacientes chagásicos em estado de emergência ou em terapia intensiva pronto socorro num hospital urbano da cidade de Buenos Aires; b) estabelecer as causas mais freqüentes de urgência no paciente com doença de Chagas neste hospital; c) definir a qual grupo ${ }^{4}$ (de acordo com a classificação do Conselho Argentino da Doença de Chagas) pertenciam os pacientes internados e a patologia predominante em cada grupo; d) analisar a relação entre a presença de cardiopatia e internação e motivo da internação e mortalidade, presença da cardiopatia e mortalidade e lugar de procedência e mortalidade; e) registrar a presença ou ausência de obra social dos internados (chagásicos e não chagásicos); f) investigar em quantos pacientes o abandono da medicação preestabelecida relaciona-se com a causa de descompensação.

\section{MATERIAL E MÉTODOS}

Os pacientes ingressados no pronto socorro do Hospital Público General de Agudos "Francisco Santojanni" da cidade de Buenos Aires (Argentina), que recebe pacientes adultos, grávidas e crianças procedentes, especialmente, de bairros periféricos da Capital Federal habitados em sua maioria pela classe social media e inclusive de baixos recursos econômicos, durante o período de janeiro de 1998 a janeiro de 1999, foram interrogados (eles e seus familiares) sobre antecedentes epidemiológicos da doença de Chagas e circunstancias de transfusão previas ou uso de drogas endovenosas. Naqueles pacientes que relataram a doença ou apresentavam epidemiologia de risco, antecedentes transfusionais, uso de drogas endovenosas ou pais com doença reconhecida ou com suspeita, foi realizado o exame sorológico completo: teste ELISA, imunofluorescência indireta (IFI) hemaglutinação indireta $(\mathrm{HAI})$. Considerou-se reativa a positividade, no mínimo, de duas das reações. Consideraram-se reativos os valores iguais ou maiores de 1/32 para IFI e HAI e a positividade do Teste ELISA. Dentre as variáveis avaliadas nos pacientes com sorologia reativa para Chagas, considerou-se: a) idade e sexo; b) procedência, c) sintomatologia de ingresso, d) presença ou ausência de cardiopatia em relação à classificação do Conselho Argentino de Doença de Chagas da Sociedade Argentina de Cardiologia ${ }^{4}$ (Tabela 1), e) relação entre a causa de internação e a presença ou 
Tabela 1 - Classificação clinicocardiológica da doença de Chagas ${ }^{4}$, segundo a Sociedade Argentina de Cardiologia.

\begin{tabular}{ll}
\hline Classificação & Sintomas \\
\hline Chagas agudo & parasitemia + \\
Chagas indeterminado & sorologia + \\
& exame físico normal \\
& estudos complementares normais (eletrocardiograma, Rx de tórax, ergometria) \\
& exame do aparelho digestivo normal \\
Chagas com cardiopatia & \\
Grupo "A" & arritmias e/ou transtorno de condução sem cardiomegalia \\
Grupo "B" & insuficiência cardíaca com cardiomegalia congestiva \\
\hline
\end{tabular}

ausência de cardiopatia chagásica, f) mortalidade e causas da mesma, g) presença ou ausência de obra social. Registrou-se, também, a quantidade de pacientes que no momento da internação abandonaram a medicação previamente prescrita pelo médico e que poderia ser a causa da provável descompensação cardíaca.

O diagnóstico definitivo para internação realizou-se mediante exaustivo interrogatório, avaliação clínica e semiológica, estudos complementares necessários para confirmar o diagnóstico definitivo (Tabela 2).

A metodologia estatística utilizada foi o teste de $\mathrm{X}^{2}$ com correção de Yates, para pôr a prova a hipótese de independência entre as variáveis contrastadas. Assim, avaliou-se: a) diagnóstico de internação e grupo evolutivo ao que pertencia o paciente (Tabela 1), b) diagnóstico de internação e mortalidade, c) grupo evolutivo ao que pertencia o paciente e mortalidade e d) procedência geográfica e mortalidade.

Tabela 2 - Estudos complementares realizados para chegar ao diagnóstico definitivo, necessários para internação.

\begin{tabular}{|c|c|}
\hline Diagnóstico & Estudos complementares \\
\hline Insuficiência cardíaca descompensada* & eletrocardiograma, radiografia de tóxax, ecocardiograma \\
\hline Síncope ${ }^{\star *}$ & $\begin{array}{l}\text { eletrocardiograma, radiogfrafia de tórax, monitor cardiológico, holter de } 24 \mathrm{hs} \text {, estudo } \\
\text { eletrofisiológico }\end{array}$ \\
\hline Acidente vascular cerebral ${ }^{*}$ & tomografia computadorizada de cérebro com e sem contraste \\
\hline Infarto agudo de miocárdio* & laboratório (enzimas cardíacas: CPK-LDH-TGO-CPKMB), eletrocardiograma \\
\hline Angina instável* & $\begin{array}{l}\text { laboratório (enzimas cardíacas: CPK-LDH-TGO-CPKMB), eletrocardiograma, } \\
\text { cineangiocoronariografia }\end{array}$ \\
\hline Insuficiência respiratória aguda** & $\begin{array}{l}\text { radiografia de tórax, cintilograma de ventilação perfusão, infectológicos, tomografia } \\
\text { computadorizada }\end{array}$ \\
\hline Doenças infecciosas & $\begin{array}{l}\text { hemoculturas, uroculturas, punção lombar para estudo de LCR, exame de esputo, } \\
\text { radiografia de tórax, ecografias, tomografia computadorizada, etc }\end{array}$ \\
\hline
\end{tabular}

\section{RESULTADOS}

Dos 1.680 pacientes internados no pronto socorro do Hospital Francisco Santojanni em estado de emergência médica, durante o período de um ano, 95 $(6 \%)$ pacientes apresentaram sorologia reativa para Chagas. A idade média destes pacientes foi de 54 anos e a distribuição por sexo foi de $43(45 \%)$ mulheres e $52(55 \%)$ homens. Não foram observadas diferenças significativas nas idades dos distintos grupos. Porém, os pacientes provenientes da Bolívia tinham tendência a ser mais jovens. A procedência dos pacientes, a idade média e a distribuição por sexo estão esquematizadas na Tabela 3 . Os três pacientes nascidos na Província de Buenos Aires tinham antecedentes de hemotransfusão.
Quanto à sintomatologia apresentada na internação, observou-se o seguinte: dispnéia em 35 pacientes, síncope em 31 pacientes, edema bimaleolar em 28 pacientes, angor em 18 pacientes, hemiplegia/hemiparesia em 8 pacientes, cefaléia intensa e vômitos em 2 pacientes. A respeito dos grupos aos quais pertenciam (Tabela 1), observou-se que dos 95 pacientes chagásicos, $13(13,7 \%)$ correspondiam ao grupo indeterminado, $10(10,5 \%)$ ao grupo de cardiopatas "A" e $72(75,8 \%)$ ao grupo de cardiopatas "B".

Os motivos da internação relacionados à presença ou à ausência de cardiopatia estão descritos na Tabela 4. A primeira causa de internação do paciente chagásico foi a síncope observada em 31 pacientes; destes, bloqueio aurículoventricular, de segundo e terceiro grau, 
Tabela 3 - Lugar de nascimento dos pacientes chagásicos internados.

\begin{tabular}{|c|c|c|c|c|c|c|}
\hline \multirow[b]{2}{*}{ País } & \multirow[b]{2}{*}{ Província } & \multirow[b]{2}{*}{ Idade média } & \multicolumn{2}{|c|}{ Sexo } & \multicolumn{2}{|c|}{ Paciente } \\
\hline & & & $\mathrm{F}$ & M & $\mathrm{n}^{\circ}$ & $\%$ \\
\hline Argentina & Santiago del Estero & 55 & 16 & 29 & 45 & 47,5 \\
\hline Bolívia & - & 37 & 4 & 8 & 12 & 12,7 \\
\hline Argentina & Tucumán & 58 & 6 & 3 & 9 & 9,5 \\
\hline Argentina & Chaco & 59 & 6 & 2 & 8 & 8,5 \\
\hline Argentina & Salta & 62 & 2 & 1 & 3 & 3,1 \\
\hline Argentina & Córdoba & 57 & 1 & 2 & 3 & 3,1 \\
\hline Argentina & Buenos Aires & 52 & 3 & & 3 & 3,1 \\
\hline Argentina & San Luis & 57 & 1 & 2 & 3 & 3,1 \\
\hline Argentina & Corrientes & 59 & 1 & 1 & 2 & 2,1 \\
\hline Argentina & Catamarca & 60 & 1 & 1 & 2 & 2,1 \\
\hline Argentina & Misiones & 48 & 1 & 1 & 2 & 2,1 \\
\hline Paraguai & - & 55 & & 2 & 2 & 2,1 \\
\hline Argentina & Jujuy & 48 & 1 & & 1 & 1,0 \\
\hline
\end{tabular}

Tabela 4 - Relação entre a causa de internação e presença de cardiopatia.

\begin{tabular}{|c|c|c|c|c|c|c|c|}
\hline \multirow[b]{2}{*}{ Causas de internação } & \multirow[b]{2}{*}{ Idade média } & \multicolumn{2}{|c|}{ Sexo } & \multirow[b]{2}{*}{ GI } & \multicolumn{2}{|c|}{$\mathrm{GC}$} & \multirow[b]{2}{*}{ Total } \\
\hline & & $\mathrm{F}$ & M & & "A" & "B" & \\
\hline Sincope & 51 & 18 & 13 & & 5 & 26 & 31 \\
\hline ICC & 54 & 10 & 18 & & & 28 & 28 \\
\hline Coronariopatia & 63 & 6 & 12 & 6 & 2 & 10 & 18 \\
\hline AVC & 61 & 4 & 4 & 1 & 2 & 5 & 8 \\
\hline EAP & 49 & 2 & 1 & 3 & & & 3 \\
\hline IRA & 57 & 3 & 2 & 1 & 1 & 3 & 5 \\
\hline Encefalite aguda & 41 & & 2 & 2 & & & 2 \\
\hline Total & 54 & 43 & 52 & 13 & 10 & 72 & 95 \\
\hline
\end{tabular}

GI: Grupo Indeterminado. GC: grupo de cardiopatas. ICC: Insuficiência cardíaca congestiva. AVC: Acidente vascular cerebral. EAP: Edema agudo de pulmão. IRA: Insuficiência respiratória aguda.

Os números indicam os distintos pacientes chagásicos colocados nos diferentes grupos.

foi obsevado em 18 pacientes e o restante (13 pacientes) apresentou quadro de taquicardia ventricular persistente. Com relação ao tratamento dos pacientes com síncope, observou-se o seguinte: 10 (42\%) foram para implantação de marcapasso definitivo mais anti-arritmicos, 7 (29\%) para colocação de marcapasso definitivo, 4 (17\%) receberam tratamento farmacológico com anti-arritmicos, exclusivamente, e $3(12 \%)$ implantaram um cardiodesfibrilador. Os 7 pacientes restantes faleceram de forma súbita quando ingressaram (1 deles com bloqueio aurículoventricular de terceiro grau que sofreu assistolia imediata com resposta negativa às manobras de ressuscitação e 6 pacientes ingressaram com taquicardia ventricular persistente que evoluiu para fibrilação ventricular; sem resposta à cardioversão elétrica e às drogas de uso habitual na ressuscitação).

A insuficiência cardíaca descompensada foi observada em 28 pacientes e constituiu na segunda causa de internação. Dos 18 pacientes ingressados com esse diagnóstico, 17 (61\% do total dos casos) faleceram, todos por insuficiência cardíaca refratária ao tratamento médico, e por se tratarem de pacientes com antecedentes de capacidade funcional III-IV, prévia à descompensação. Esses pacientes, habitualmente, apresentam prognóstico obscuro, sendo prognóstico escuro é por isso que a mortalidade nestas circunstâncias foi elevada. A terceira causa de internação, observada em 18 pacientes, correspondeu ao quadro coronário em presença da doença de Chagas, a saber: 8 pacientes por infarto agudo do miocárdio confirmado pela clínica, eletrocardiograma e aumento enzimático ( 5 tratados com estreptoquinasa e $3 \mathrm{com}$ nitroglicerina por estarem fora do período); 10 pacientes com angina instável com mudanças eletrocardiográficas ( 9 pacientes receberam tratamento médico e 1 precisou de cirurgia mediante ponte aortocoranário nos dias posteriores). Deve-se destacar que nos 10 pacientes com quadro de angina instável, em 9 foi confirmada a origem ateroeclerótico da doença por cineangiocoronariografia.

Todos os pacientes que sofreram alteração do quadro coronário apresentaram como antecedentes a associação de dois ou mais fatores de risco coronário. Dos 8 pacientes que sofreram acidentes vasculares cerebrais (AVC), 6 apresentaram AVC hemorrágico em relação a quadros hipertensivos e 2 AVC isquêmico em pacientes com cardiopatia dilatada em presença de fibrilação auricular e sem tratamento anticoagulante. Outra causa de internação correspondeu ao quadro de edema agudo de pulmão, de origem hipertensiva, 
observada em 3 pacientes chagásicos em período indeterminado. Dos 5 pacientes que ingressaram com quadro de insuficiência respiratória aguda, 3 apresentaram quadro de tromboembolismo pulmonar (TEP) e 2 quadro de tuberculose em estado grave. Dos 3 pacientes com quadro de TEP, todos apresentavam miocardiopatia dilatada, 2 com fibrilação auricular sem tratamento anticoagulante e 1 deles com ritmo sinusal com presença de terreno varicoso importante e também sem tratamento anticoagulante.

Finalmente, deve-se destacar 2 casos de encefalite aguda por reagudização da doença de Chagas em pacientes com síndrome da imunodeficiência adquirida (SIDA). Ambos os pacientes eram chagásicos em período indeterminado, com antecedentes de consumo de drogas endovenosas. Nos dois casos diagnosticou-se, também, presença do T. cruzi no líquido cefalorraquídeo e ambos faleceram, a pesar do tratamento com benznidazol, possivelmente pelo avançado estado da doença no momento da consulta.

Com relação à assistência social, observou-se uma diferença significativa $(p<0,01)$ entre pacientes chagásicos e não chagásicos (Tabela 5).

O abandono da medicação prévio, à descompensação, observou-se em 28 (29\%) dos 95 pacientes, sendo que em $25(89 \%)$ pacientes, a principal causa foi a falta de meio econômico para adquirir os medicamentos e por decisão própria nos $3(11 \%)$ restantes. Entre os 28

Tabela 5 - Assistência Social a pacientes chagásicos e não chagásicos atendidos no pronto socorro.

\begin{tabular}{|c|c|c|c|c|c|}
\hline \multirow[b]{2}{*}{ Internados } & \multirow[b]{2}{*}{ Total } & \multicolumn{2}{|c|}{ Com Assistência Social* } & \multicolumn{2}{|c|}{ Sem Assistência Social } \\
\hline & & $n^{\circ}$ & $\%$ & $\mathrm{n}^{\circ}$ & $\%$ \\
\hline Chagásicos & 95 & 7 & 7 & 88 & 93 \\
\hline Não chagásicos & 1.585 & 395 & 25 & 1190 & 75 \\
\hline
\end{tabular}

pacientes que abandonaram a medicação, a principal causa de internação foi a insuficiência cardíaca descompensada (19), seguida de síncope de origem arrítmico (5), abandono de drogas em pacientes com antecedentes de angina instável (2), interrupção do tratamento anti-hipertensivo que teve como conseqüência a presença de EAP (1) e por abandono do tratamento anticoagulante que favoreceu o aparecimento de AVC isquêmico (1).

No que se refere à mortalidade, $27(28 \%)$ pacientes faleceram no pronto socorro durante o transcurso da internação, como conseqüência da descompensação hemodinâmica. As causas de morte foram choque cardiogênico em 17 (48\%) pacientes, forma súbita em 7 (41\%), encefalite aguda em 2 (7\%) e TEP em 1 (4\%) paciente.

Observou-se dependência altamente significativa entre o motivo da internação e o grupo evolutivo dos pacientes (Tabela 6) com um valor de $\chi^{2}=101,56(p<0,01)$. O que indica que a internação esteve significativamente associada à presença de cardiopatia. A presença de insuficiência cardíaca congestiva, síncope, AVC, coronariopatia e insuficiência respiratória aguda demonstra uma associação significativa com a cardiopatia (Grupos A e B).

Tabela 6 - Relação entre os grupos, segundo sua evolução e os motivos de internação.

\begin{tabular}{lcccccccc}
\hline Grupos & Insuficiência cardíaca & Síncope & AVC & Coronariopatia & EAP & IRA & Encefalite aguda & Total \\
\hline Indeterminado & - & - & 1 & 6 & 3 & 1 & 2 & 13 \\
Grupo "A" & - & 2 & 2 & 4 & - & 2 & - & 10 \\
Grupo "B" & 28 & 29 & 5 & 8 & - & 2 & - & 72 \\
\hline Total & 28 & 31 & 8 & 18 & 3 & 5 & 2 & 95 \\
\hline
\end{tabular}

AVC: acidente vascular cerebral. EAP: edema agudo de pulmão. IRA: insuficiência respiratória aguda.

A dependência entre mortalidade e o motivo da internação (Tabela 7) também resultou significativa, com um valor de $\chi^{2}=40,9(p<0,01)$. Isto significa que a presença de patologias graves, como insuficiência cardíaca refratária ao tratamento médico, síncope (fundamentalmente de origem arrítmico, especialmente, por taquicardia ventricular persistente que deriva em fibrilação ventricular), tromboembolismo pulmonar e encefalite esta associada de forma significativa com a mortalidade. Do mesmo modo, foi estatisticamente significativa a dependência entre o grupo a que pertenciam e a mortalidade (Tabela 8 ), com um valor de $\chi^{2}=9 \quad(p<0,05)$. O achado de cardiopatia, fundamentalmente dilatada no grupo " $B$ " esteve significativamente associado à mortalidade e somente se apresentou no período indeterminado; produto da reativação da doença de Chagas no contexto de uma doença grave como SIDA.

Finalmente, observou-se também uma dependência significativa entre a procedência dos pacientes com relação à mortalidade (Tabela 9 ), com um valor de $\chi^{2}=31,65(p<0,01)$. 
Tabela 7- Relação entre mortalidade e motivo de internação.

\begin{tabular}{lcccccccc}
\hline Mortalidade & Insuficiência cardíaca & Síncope & AVC & Coronariopatia & EAP & IRA & Encefalite aguda & Total \\
\hline Falecido & 17 & 7 & - & - & - & 1 & 2 & 27 \\
Vivo & 12 & 24 & 8 & 17 & 3 & 4 & - \\
\hline Total & 29 & 31 & 8 & 17 & 3 & 5 & 68 \\
\hline
\end{tabular}

AVC: acidente vascular cerebral. EAP: edema agudo de pulmão. IRA: insuficiência respiratória aguda.

Tabela 8 - Relação entre grupo evolutivo e mortalidade.

\begin{tabular}{lcccc}
\hline & & \multicolumn{2}{c}{ Grupos } & \\
\cline { 3 - 4 } Mortalidade & Indeterminados & "A" & "B" & Total \\
\hline Falecido & 2 & - & 25 & 27 \\
Vivo & 11 & 10 & 47 & 68 \\
\hline Total & 13 & 10 & 72 & 95 \\
\hline
\end{tabular}

Tabela 9 - Relação entre a procedência dos pacientes e mortalidade.

\begin{tabular}{lccc}
\hline Procedência & Falecido & Vivo & Total \\
\hline Santiago del Estero & 11 & 34 & 45 \\
Bolívia & 5 & 7 & 12 \\
Tucumán & 3 & 6 & 9 \\
Chaco & 2 & 6 & 8 \\
Salta & 2 & 1 & 3 \\
Córdoba & - & 3 & 3 \\
Buenos Aires & 1 & 2 & 3 \\
San Luis & - & 3 & 3 \\
Corrientes & 1 & 1 & 2 \\
Catamarca & 1 & 1 & 2 \\
Misiones & - & 2 & 2 \\
Paraguai & - & 2 & 2 \\
Jujuy & 1 & - & 1 \\
\hline Total & 27 & 68 & 95 \\
\hline
\end{tabular}

Desta forma, o fato de proceder do Norte da Argentina (especialmente de Santiago del Estero, Tucumán, Chaco e Salta) ou de países fronteiriços como Bolívia demonstrou ter uma associação significativa com a mortalidade.

\section{DISCUSSÃO}

A prevalência de ingresso de pacientes com doença de Chagas em estado de emergência no pronto socorro de um hospital urbano da Cidade de Buenos Aires foi de $6 \%$, uma percentagem elevada se considerarmos que os dados oficiais divulgam 2,3\% de prevalência para esta cidade e $2,5 \%$ para a Província de Buenos Aires ${ }^{21}$. Este fato encontrase intimamente relacionado com a notável migração interna de províncias endêmicas empobrecidas e o incremento de imigração estrangeira, fundamentalmente, da Bolívia e do Paraguai $^{2}$.

Com relação aos motivos de internação em estado de emergência, deve-se destacar que a síncope e a insuficiência cardíaca foram as principais causas de internação.

$\mathrm{Na}$ primeira delas, a mortalidade estava mais associada à causa arrítmica que aos transtornos de condução graves. Provavelmente, está relacionada com o pouco tempo que a causa arrítmica deixa para que os profissionais tomem uma ação eficaz e porque muitas vezes sua transformação reiterada de taquicardia ventricular em fibrilação ventricular converte o quadro inicial de síncope em morte súbita. Nestes casos, as manobras de ressuscitação tornam-se inúteis, sobretudo em pacientes dilatados e com má fração de ejeção. A terceira causa de internação, sem dúvida fez reavaliar as possibilidades do desenvolvimento de coronariopatia em pacientes com Chagas. Durante muitos anos, alguns trabalhos no Brasil demonstraram que a presença de coronariopatia ateroesclerótica era de rara observação no paciente chagásico crônico ${ }^{3} \mathrm{e}$ os poucos casos de infarto agudo de miocárdio eram produto de fenômenos embólicos. Isto ademais era abalado pelos escassos achados de infarto agudo de miocárdio em milhares de autópsias realizadas em pacientes com Chagas ${ }^{14}$. Não obstante, a probabilidade de um diâmetro maior das coronárias, produto da desnervação parassimpática com modificações do tono das mesmas, o que evitaria o desenvolvimento de coronariopatia, contrapõe-se com a presença de fatores de risco coronário que podem originar o início de mecanismos aterogênicos que resultem na obstrução de artérias coronárias. Este 
último fato como conseqüência da forma de vida em área urbana que, somado ao desarraigo vivido pelo paciente ao abandonar seu lugar de origem, gera o estresse típico das grandes urbes. Estas mudanças na conduta que levam a uma alta prevalência de sedentarismo, obesidade, hipercolesterolemia, tabagismo, hipertensão arterial e diabetes, aumentam o índice de risco.

Observaram-se alguns quadros de complicações e morte gerados por tromboembolismo pulmonar e acidentes cerebrovasculares isquêmicos ${ }^{12}{ }^{16} \mathrm{em}$ pacientes com indicação de anticoagulação e os que não a recebiam. Acontece que em muitos casos esta indicação médica não se realiza por falta de recursos econômicos, analfabetismo e migrações por razões laborais, o que faz que o paciente chagásico não realize os controles hematológicos periódicos, sendo nestes casos "pior o remédio que a doença". Finalmente, o incêndio da doença de Chagas ${ }^{23}$ em presença da SIDA é uma patologia provável na atualidade com quadros de encefalite o miocardite muitas vezes mortais e que põe em evidência a alta patogenicidade da reinfecção endógena em presença da imunodepressão adquirida ${ }^{17}$.

A maior proporção de internados registrou-se no grupo de cardiopatas, fundamentalmente nos dilatados. As internações em pacientes em período indeterminado deveram-se à descompensação de doenças associadas à Chagas (ex. coronariopatias, edema agudo de pulmão hipertensivo, etc.) e em duas oportunidades a reagudização da doença de Chagas em pacientes com SIDA. As principais causas de internação no grupo de cardiopatas não dilatados "A" deveram-se à descompensação de doenças associadas à Chagas e as causas de internação relacionadas diretamente com esta doença obedeceram a quadros de síncopes. Nos cardiopatas dilatados "B", a principal causa de internação foi a síncope, seguida da insuficiência cardíaca descompensada. Isto se relaciona com a análise multivariada, de 2.260 pacientes chagásicos ${ }^{22}$, que demonstrou uma associação estatisticamente significativa no achado da dispnéia devido à insuficiência cardíaca congestiva e síncope com presença de dano miocárdico. Independente da doença de Chagas, alguns estudos epidemiológicos internacionais demonstraram que em torno do $3 \%$ das consultas nas salas de plantão devem-se a quadros de síncope ${ }^{25}$ e é a causa de 1 a $6 \%$ de ingressos na sala de internação geral ${ }^{11}$. Neste caso, na doença de Chagas a síncope é a principal causa de consulta no plantão hospitalar. Coronariopatia foi observada com maior tendência no grupo de cardiopatas "B".

$A$ assistência social significativamente menor nos pacientes chagásicos com relação à população geral estava relacionada com dois fenômenos: a) pobreza extrema dos chagásicos e b) falta de trabalho destes pacientes, seja por incapacidade física produto da doença ou por discriminação laboral, como já foi demonstrado em outro estudo ${ }^{21}$, pelo fato de apresentar sorologia reativa para Chagas. Desta forma, o paciente com Chagas não tem acesso à medicina privada, com a possibilidade de realizar os exames mais complexos e caros, como o implante de marcapasso, cardiodesfibriladores implantáveis, etc, o que obriga o governo a subsidiar estes exames e terapêuticas através da rede pública de saúde, ocasionando muitas vezes demoras que colocam em risco a vida do paciente. Este contexto associa-se, também, a um maior abandono da medicação o que condiciona à descompensação hemodinâmica e, por conseguinte, internação em estado de emergência médica.

Com relação à mortalidade dos pacientes com sorologia reativa para Chagas, não se observaram diferenças a respeito dos achados de outros autores, sendo por ordem de aparecimento: 1) insuficiência cardíaca descompensada, 2) morte súbita, 3) encefalite aguda em pacientes com Chagas e SIDA e 4) tromboembolismo pulmonar. A série de Dias ${ }^{5}$ demonstrou como causas de morte: 1) morte súbita, 2) insuficiência cardíaca, 3) encefalite aguda em pacientes com Chagas e SIDA, e 4) tromboembolismo pulmonar. Por outro lado, Espinosa e $\mathrm{col}^{8}$, acharam como principal causa de mortalidade a insuficiência cardíaca descompensada, seguida de morte súbita e por tromboembolismo pulmonar. No presente trabalho, deve destacar-se a existência da relação estatisticamente significativa entre mortalidade e: 1) presença de cardiopatia dilatada e 2) procedência geográfica do Norte da Argentina (Santiago del Estero e Tucumán) e Bolívia, em pacientes jovens (entre 37 e 58 anos) que não dependeu da associação de outras doenças cardiológicas.

Lamentavelmente, não se encontraram dados de outras pesquisas similares na Argentina (nem no hospital onde se realizou o estudo) que puderam ser comparados e analisados a respeito deste trabalho.

Em resumo, as causas de emergência e mortalidade dos pacientes chagásicos nas salas de terapia intensiva de plantão das grandes urbes como a Cidade de Buenos Aires, demonstram que a evolução natural da cardiopatia, cujo início se produz nas áreas endêmicas com condições de vida extremas, agrava-se quando somados os fatores de risco próprio da vida estressante do habitat urbano. Portanto, a esperança de melhorar a qualidade de vida na cidade não evita o desenlace final de uma cardiopatia chagásica, de origem infecciosa, que é afetada pelas doenças degenerativas da civilização, condicionando a descompensação à morte nos pronto socorro.

\section{AGRADECIMENTOS}

A todos os pacientes chagásicos que, apesar do seu estado de saúde, colaboraram com boa predisposição para fazer possível o trabalho científico. 


\section{REFERÊNCIAS BIBLIOGRÁFICAS}

1. Apt W, Arribada A, Aguilera X, Sandoval J. Cardiomiopatía chagásica y Zimodemos de T. cruzi en Chile. Boletín de la Oficina Sanitaria Panamericana 104:450-461, 1998.

2. Auger S, Storino R. Realidad social y laboral del paciente chagásico. Revista Argentina de Medicina 1:7-10, 1998.

3. Bestetti RB, Finzi LA, Oliveira JSM. Chronic Chagas heart disease presenting as an impending myocardial infarction: A case favoring the neurogenic pathogenesis concept. Revista Clínica Cardiológica 10:368-370, 1987.

4. De Rosa M, Storino R, Mitelman J, Leguizamon Palumbo J, Madoery R, losa D, Costa C, Auger S, Romero Villanueva H. Enfermedad de Chagas: clasificación clínico-cardiológica. Revista Argentina de Cardiología 66:243-244, 1998.

5. Dias JCP. Doença de Chagas em Bambui, Minas Gerais, Brasil. Estudo clínico epidemiológico a partir da fase aguda entre $1940 \mathrm{e}$ 1982. Universidade Federal de Minas Gerais, Belo Horizonte, 1982.

6. Dias JCP. Etiopatogenia e historia natural da doença de Chagas humana. Revista de Patología Tropical 14:17-29, 1985.

7. Dias JCP, Lugones SH. Chagas. Curso de enfermedades tropicales regionales y emergentes. Universidad Católica de Salta. Módulo II:13-141, 1999.

8. Espinosa R, Carrasco H, Belandria F, Fuenmayor A, Molina C, Martinez G. Life expentacy analysis in patients with Chagas disease: Prognosis after one decade (1973-1983). International Journal of Cardiology 8:45-56, 1985.

9. Going JC, Borda ES, Auger S, Storino R, Sterin-Borda L. Cardiac M2 muscarinic cholinoceptor activation by human chagasic autoantibodies: association with bradycardia. British Heart Journal 82:273-278, 1999

10. Kaplan D, Ferrari I, Bergami P, Mohler E, Levitus G, Chiale P, Levin M. Los anticuerpos antiproteínas ribosomales "P" estimulan al receptor $B$ adrenérgico en la enfermedad de Chagas, pero no en el Lupus Eritematoso Sistémico. Revista Argentina de Cardiología 66:127-138, 1998.

11. Kapoor W. Diagnostic evaluation of syncope. American Journal of Medicine 91:91-106, 1991.

12. Lopes ER, Marquez JO, Costa Neto B, Menesez AAC, Chapadeiro E. Associação entre acidentes vasculares encefálicos e doença de Chagas. Revista da Sociedade Brasileira de Medicina Tropical 24:101-104, 1991.
13. Macedo FO. Insuficiência de exposição e reinfecção na evolução da doença de Chagas (Estudo longitudinal de 5 anos). Revista de Patología Tropical 5:33-116, 1973.

14. Oliveira JSM, Araujo RRC, Navarro MA, Mucillo G. Cardiac thrombosis and thromboembolism in chronic Chagas. American Journal of Cardiology 52:147-151, 1983.

15. Organización Mundial de la Salud. Control de la Enfermedad de Chagas. Serie de Informes Técnicos 811, OMS. Genebra, 1991.

16. Pitella JEH. Ischemic cerebral changes in the chronic chagasic cardiopathy. Arquivos Medicos de Psiquiatria 42:105-115, 1984

17. Rocha A, Meneses ACO, Silva AM, Ferreira M, Nishioka SA, Burgarelli MKN, Almeida E, Metze K, Turcato Jr G, Lopes ER. Pathology of patients with Chagas disease and acquired immunodeficiency sydrome. The American Journal of Tropical Medicine and Hygiene 50:261-268, 1994

18. Ruiz AM. La enfermedad de Chagas, una parasitosis americana Centro de referencia internacional del diagnóstico de la enfermedad de Chagas Fatala Chabén. Publicación del Instituto "Fatala Chabén", Buenos Aires, Argentina, 1998.

19. Storino R. Evolución natural y fisiopatogenia de la enfermedad de Chagas humana. Tesis de doctorado, Universidad Nacional de la Plata, Buenos Aires, Argentina, 1984.

20. Storino R, Auger S. Jörg M. Cuál es la realidad de la enfermedad de Chagas en Argentina y América? Revista Medicina de Buenos Aires 57:513-516, 1997.

21. Storino R, Auger S, Jörg M, Leguizamón Palumbo J, Urrutia MI. Discriminación social y marginalidad laboral del paciente chagásico Revista Norte-Nordeste do Coraçao 4/5:8-22, 1997.

22. Storino R, Auger S, Wojdyla D, Urrutia MI, Jörg M. Análisis estadístico multivariado de la enfermedad de Chagas en 2260 pacientes. Premio "Fundación Pedro Cossio". Otorgado por la Sociedad Argentina de Cardiología. Revista Argentina de Cardiología 66:17-43, 1998.

23. Storino R, Jörg M, Auger S. Manual práctico de atención médica del paciente chagásico. Editora Ediprof. Masson-Doyma, Argentina, 2002.

24. Storino R, Milei J. Enfermedad de Chagas. Editora Mosby-Doyma, Argentina, 1994.

25. Stults BM, Gandolfi RG. Diagnostic evaluation for syncope. West Journal of Medicine 144:234, 1986 